\title{
Editorial
}

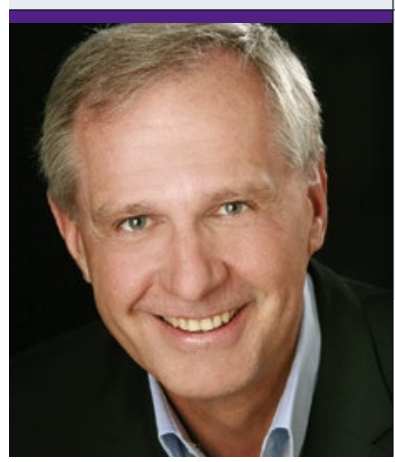

"Im Silicon Valley ist man sich inzwischen einig,

dass, the next big thing' das Thema Gesundheit ist."

Prof. Dr. med. Bernd Kleine-Gunk

Leiter der Gynäkologie an der Schön Klinik Nürnberg/Fürth und Präsident der

Deutschen Gesellschaft für Prävention und Anti-Aging Medizin (GSAAM)

\section{Schöne neue Medizinwelt}

$\mathrm{V}$ erglichen mit den teilweise atemberaubenden Innovationen, denen manche Berufszweige in den letzten Jahren ausgesetzt waren, hat sich im Arbeitsalltag des niedergelassenen Gynäkologen nur relativ wenig verändert. Für den seit über 50 Jahren durchgeführten Pap-Abstrich benutzen wir inzwischen eine Bürste statt einen Wattetupfer. Die seit 30 Jahren bestehende Transvaginalsonografie ist inzwischen zwar höher auflösend, sie wird prinzipiell aber immer noch so durchgeführt wie schon 1985. Die seit 60 Jahren existierenden oralen Kontrazeptiva gibt es jetzt auch mit natürlichen Östrogenen. Und mit vielen neuen lustigen Namen für alte Präparate. So richtig revolutionär ist das alles nicht.

Das könnte sich bald ändern und zwar aus folgendem Grund: Inzwischen drängen ganz neue Akteure auf den Medizinmarkt. Traditionell waren hier zwei Branchen dominierend. Die Pharmaindustrie entwickelte neue Medikamente, die Medizintechnik neue Geräte. Nun kommt ein völlig neuer Mitbewerber hinzu: Die äußerst innovationsfreudige Informationstechnologie.

Im Silicon Valley ist man sich inzwischen einig, dass „the next big thing“ das Thema Gesundheit ist. Branchenführer Apple wendet sich mit immer mehr Produkten an die „,self-tracker“, die jegliche Körperfunktion - vom Blutdruck über das Schlafverhalten bis zum Bewegungsmuster - messen, speichern und analysieren. Der Suchmaschinenbetreiber Google, inzwischen das reichste und einflussreichste Unternehmen der Welt, hat mit Calico (California Life Corporation) eines seiner berühmten MoonshotsProjects gestartet, also jene Projekte, welche die Welt verändern sollen. Ziel von Calico ist nicht mehr und nicht weniger als die Behandlung des biologischen Alterns. Und IBM, der Spezialist für Großrechner, sieht als eines seiner erfolgversprechendsten Produkte inzwischen „Dr. Watson“, ein Computerprogramm, das ständig mit den neuesten medizinischen Publikationen und sämtlichen Leitlinien gefüttert wird und bezüglich der Diagnosestellung längst jede Unikoryphäe in den Schatten stellt.

Es gibt wohl nur wenig Zweifel, dass dieses massive Engagement führender Unternehmen aus dem IT-Bereich das klassische medizinische Feld völlig umkrempeln wird. Der traditionell eher wenig technikaffine niedergelassene Arzt wird sich auf diese Entwicklungen einstellen und dazulernen müssen.

Die Frage ist nicht, ob „Dr. Watson“ kommt oder nicht. Er kommt mit Sicherheit. Die Frage ist lediglich, ob wir ihn zu einem kompetenten, elektronischen Assistenzarzt und Zuarbeiter machen. Oder ob das elektronische Diagnosegenie vielen seiner realen Kollegen den Job kosten wird.

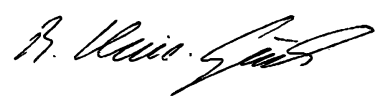

